\title{
Über Angst in der Literatur
}

Tilmann Köppe

Angst ist ein Phänomen von allgemeinmenschlicher Bedeutung, und so ist nicht verwunderlich, dass Angst in ihren unterschiedlichen Ausprägungen auch in der sschönen< Literatur behandelt wird. Dieser Beitrag betrachtet einige der Weisen, wie das geschehen kann: Die Beispiele entstammen der Lyrik sowie der Erzählliteratur (ohne dass damit nahegelegt werden sollte, dass Angst nicht auch in anderen Genres oder Gattungen Gegenstand ist). Diskutiert wird zunächst (und notwendig exemplarisch), wie Angst im literarischen Text dargestellt werden kann, anschließend wende ich mich den Effekten oder Funktionen solcher Darstellungen zu. Das Augenmerk gilt dabei dem Ausdruck (der Expressivität) von Angst, der Literatur als trigger von Angst sowie einer Diskussion der Frage, wie sich die Darstellung von Angst in der Literatur mit deren Charakter als sschöner Literatur und Quelle ästhetischen Vergnügens verträgt.

Überlegungen zur >literarischen Angst kommen um eine (und sei es knappe) Gegenstandsbestimmung nicht herum: Was ist unter Angst (hier) zu verstehen? In der philosophischen Anthropologie, in Biologie oder Psychologie sind unterschiedliche Bestimmungen des Begriffs >Angst vorgeschlagen worden. Das Spektrum der Bestimmungen ist breit; es schließt evolutionsbiologische Theorien ein, denen zufolge Angst ein "Stadium" ist, in dem ein Säugetier "kein angemessenes Verhaltensprogramm parat hat $"{ }^{1}$ und philosophische Theorien, die Angst »als Genese der Subjektivität« verstehen möchten. ${ }^{2}$ Im Sinne einer etwas genaueren Eingrenzung werden Angstphänomene oftmals dem genus proximum der Stimmungen zugeordnet. Stimmungen lassen sich verstehen als Dispositionen einer Person, ihre Gesamtsituation in bestimmter, affektiv getönter Weise wahrzunehmen, zu erfahren oder zu beurteilen:

Somewhat as the entire ship is cruising south-east, rolling, or vibrating, so the entire person is nervous, serene or gloomy. His own corresponding inclination will be to describe the whole

\footnotetext{
1 Karl Eibl,Von der biologischen Furcht zur literarischen Angst. Ein Vertikalschnitt, in: KulturPoetik 12 (2012), 155-186, hier 160.

2 So Hinrich Fink-Eitel in einer Exegese von Kiergegaards richtungsweisenden Überlegungen zu Angst (Hinrich Fink-Eitel, Angst und Freiheit. Überlegungen zur philosophischen Anthropologie, in: Zur Philosophie der Gefühle, hg. v. Hinrich FinkEitel/Georg Lohmann, Frankfurt a.M. 1993, 57-88, hier 77).
} 
world as menacing, congenial, or grey. If he is jovial, he finds everything jollier than usual; and if he is sulky, not only his employer's tone of voice and his own knotted shoe-lace seem unjust to him, but everything seems to be doing him injustices. ${ }^{3}$

Für die Stimmung der Angst ist charakteristisch, dass die Welt (sozusagen im Ganzen) als bedrohlich erfahren wird. Das unterscheidet die Angst (als eine Stimmung) von Emotionen, für die charakteristisch ist, dass sie stets einen konkreten Gegenstand haben. Oft gegenübergestellt werden die Stimmung Angst und die Emotion Furcht: Diese gilt etwas Bestimmtem, d.h., wer sich vor etwas fürchtet, kann stets angeben, was es ist, wovor er oder sie sich fürchtet. Das ist bei Angst anders; wer sich im Zustand der Angst befindet, kann keinen einzelnen Gegenstand benennen, vor dem er oder sie Angst hat und der als Ursache der Angst gelten kann - vielmehr erscheint eben alles als (ggf. diffus) `bedrohlich ${ }^{4}{ }^{4}$ Entsprechend kann man sich von Angst auch nicht befreien, indem man einen bestimmten Gegenstand oder Sachverhalt unschädlich macht oder vermeidet (so wie man um einen Hund einen Bogen machen kann, vor dem man sich fürchtet). Es liegt auf der Hand, dass der Gefühlston der Angst (wie es ist bzw. sich anfühlt, Angst zu haben) dominant negativ (unangenehm) ist.

Fragt man nun nach der literarischen Repräsentation von Angst, so liegt es zunächst einmal nahe, in den Blick zu nehmen, wie literarische Texte davon handeln können, dass jemand Angst hat. Stellt die Literatur jemandes Angst dar, so gibt es eine Reihe systematisch unterscheidbarer Möglichkeiten, wie dies geschehen kann. ${ }^{5}$

Eine erste Weise ist die explizite (direkte) Charakterisierung einer Figur. So in Schillers Ballade »Die Bürgschaft» (1799):

Und die Angst beflügelt den eilenden Fuß,

Ihn jagen der Sorge Qualen,

Da schimmern in Abendrots Strahlen

Von ferne die Zinnen von Syrakus,

Und entgegen kommt ihm Philostratus,

Des Hauses redlicher Hüter,

3 Gilbert Ryle, The Concept of Mind, Chicago [1949] 2002, 100.

4 Unsere Sprache verschleiert die hier übernommene Unterscheidung zwischen der auf einen Gegenstand gerichteten Emotion Furcht und der ungerichteten Stimmung Angst insofern als Aussagen wie `Ich habe Angst vor der Prüfungく durchaus wohlgeformt sind.

5 Es gibt noch weitere Zusammenhänge, vgl. die Übersicht in Tilmann Köppe, Lyrik und Emotionen, in: Zeitschrift für Germanistik 22 (2012), 374-387. 
Der erkennet entsetzt den Gebieter: ${ }^{6}$

Hier ist explizit die Rede davon, Möros werde von seiner Angst zur Eile angetrieben. Meint Schiller hier die Furcht des Möros vor einem bestimmten Ereignis: der Hinrichtung des Freundes an seiner statt etwa? Vielleicht. Es könnte aber sein, dass Möros zusätzlich zu seiner Furcht vor dem Eintreffen des konkreten Ereignisses - das ihm quälende "Sorge « bereitet - auch von (allgemeiner) Angst in unserem Sinne ergriffen ist; denn in der Tat dürfte ihm in seiner Situation alles bedrohlich erscheinen, insofern alles, was ihm begegnet, das Potenzial hat, sein Schicksal zu besiegeln: Schafft er es rechtzeitig zurück nach Syrakus, droht ihm der eigene Tod; schafft er es nicht, droht der Stellvertretertod des Freundes. Die Nennung von sowohl "Sorge« (um das Schicksal des Freundes) als auch "Angst" (als umfassender Stimmung) wäre mithin kein bloßer Pleonasmus, ${ }^{7}$ sondern markierte eine semantische Differenz.

Neben der solchermaßen expliziten Charakterisierung gibt es die indirekte (implizite) Charakterisierung einer Figur als ängstlich. Wesentlicher Unterschied zum erstgenannten Verfahren ist, dass wir erst erschließen müssen, dass die Figur (wohl) Angst hat. In Annette von Droste-Hülshoffs Ballade "Der Knabe im Moor" (1842) heißt es:

Fest hält die Fibel das zitternde Kind

Und rennt als ob man es jage;

Hohl über die Fläche sauset der Wind -

Was raschelt drüben am Haage?

Das ist der gespenstige Gräberknecht,

Der dem Meister die besten Torfe verzecht;

$\mathrm{Hu}, \mathrm{hu}$, es bricht wie ein irres Rind!

Hinducket das Knäblein zage. ${ }^{8}$

Hier gibt es zwar auch eine explizite Benennung des Zustands, in dem das Kind sich befindet ("zage«), aber noch deutlicher wird die Charakterisierung des Jungen durch indirekte sprachliche Mittel. So verweist bereits das Zittern des Kindes auf seinen Erregungszustand, ebenso das Festhalten der "Fibel«, insofern man dabei an ein Festklammern denken mag; Rennen und Hinducken sind typische

\footnotetext{
6 Friedrich Schiller, Sämtliche Werke, Bd. 1, hg. v. Peter-André Alt/Albert Meier/ Wolfgang Riedel, München 2004, 355.

7 Da Schiller hier in einer Strophe von "Angst", "Sorge" und Entsetzen ("entsetzt") spricht, kann man an das Stilmittel des Pleonasmus denken, in dem ein Phänomen durch sprachliche Redundanz hervorgehoben wird.

8 Annette von Droste-Hülshoff, Historisch-kritische Ausgabe, hg. v. Winfried Woesler, Bd. I,1, Gedichte zu Lebzeiten, bearb. v. Winfried Theiss, Tübingen 1985, 67.
} 
Furcht- oder Angstreaktionen in Situationen, in denen physische Gefahr droht (wenngleich diese hier, angsttypisch, unspezifisch zu sein scheint). Auch die Situation ist beschrieben als derart, dass sie Angst hervorrufen mag: »Hohl über die Fläche sauset der Wind", etwas Unbekanntes "raschelt" in der Nähe, und sogleich geht des Jungen Fantasie mit ihm durch und er vermutet Schauerlichstes als Ursache.

Droste-Hülshoffs "Der Knabe im Moor" ist auch insofern interessant, als hier neben der direkten und indirekten Charakterisierung der Figur als ängstlich noch ein weiterer Zusammenhang von Literatur und Angst deutlich wird. Die bereits zitierte Passage zeigt diesen Zusammenhang ebenso wie die folgende:

Vom Ufer starret Gestumpf hervor,

Unheimlich nicket die Föhre,

Der Knabe rennt, gespannt das Ohr,

Durch Riesenhalme wie Speere;

Und wie es rieselt und knittert darin!

Das ist die unselige Spinnerin,

Das ist die gebannte Spinnlenor',

Die den Haspel dreht im Geröhre!?

Die Verse kombinieren einerseits die direkte Charakterisierung der Figur ("gespannt das $\mathrm{Ohr}$ " etc.) und andererseits deren indirekte Charakterisierung als ängstlich (vgl. wiederum das Rennen des Knaben etc.). Besonders charakteristisch ist für dieses Gedicht eine bestimmte Weise der indirekten Charakterisierung. Die Verse repräsentieren nämlich, wie der Knabe das Moor wahrnimmt, d.h., sie geben wieder, wie sich das Moor dem Knaben darstellt. Die Föhre snickt` (personifiziert) > unheimlich`, Schilfgräser erscheinen ihm in seiner Angst als "Riesenhalme wie Speere", das "Gestumpf" scheint ihn sanzustarren ihm (wie schon das in der Strophe zuvor zitierte $>$ Rascheln $\varsigma$ ) auf ein gespenstisches Wesen zurückzuführen zu sein. ${ }^{10}$ Damit sind diese Verse auf mehrfache Weise smotiviert` (d.h., sie erfüllen mehr als nur eine künstlerische Funktion): Neben der indirekten Charakterisierung der Figur (als ängstlich) dienen sie dem Ausdruck von Angst.

\footnotetext{
9 Ebd.

${ }^{10} \mathrm{Vgl}$. dazu die Beobachtungen in Mateusz Cwik, Wie eine gespenstige Melodey. Poetik der Wahrnehmung im Gedicht Der Knabe im Moor von Annette von Droste-Hülshoff, in: Droste-Jahrbuch 10 (2013/2014), 261-280 (dort dann allerdings in eine andere, spoetologischer Lesart mündend).
} 
Der Kunstphilosoph Robert Stecker hat die These vertreten, eine besondere Leistung der Literatur liege darin, expressiv zu sein (d.h. ein Gefühl ausdrücken zu können), indem sie uns die Welt in just jener `Tönung` anschaulich vor Augen stelle, die dem sintentionalen Gehalt eines Gefühls entspreche. Zugrunde liegt hier die These, dass die Identität einer bestimmten Emotion (also das, was dafür sorgt, dass ein Gefühlszustand ein Exemplar einer bestimmten Emotion ist) wesentlich von der Weise abhängt, in der die Person ihre Umwelt wahrnimmt bzw. geistig repräsentiert: "intentional components are what typically make an emotion the kind it is " ${ }^{11}$ Das können wir leicht auf die Stimmung der Angst übertragen: Eine Person hat nur dann Angst, wenn sie disponiert ist, die Welt als bedrohlich wahrzunehmen; die geistige Repräsentation der Umwelt als bedrohlich ist wesentlich für Angst (s.o.). Stecker zufolge können Gedichte Gefühle ausdrücken, indem sie zeigen, wie jemand, der das fragliche Gefühl hat, die Welt sieht, d.h., wie er oder sie die Welt geistig repräsentiert. Bringt ein literarischer Text jemandes Angst in diesem Sinne zum Ausdruck (indem gezeigt wird, wie sich die Welt jemandem darbietet, der Angst hat), so ist das sowohl etwas anderes als eine Beschreibung der Figur als ängstlich als auch etwas anderes als eine Beschreibung der in Rede stehenden Weise der Weltwahrnehmung.

Stecker betont, dass der literarische Gefühlsausdruck nicht nur durch das Was der Darstellung (also das explizit Gesagte), sondern auch durch das Wie der Darstellung (also rformaler Gestaltungsmittel) geschaffen werde. Metrik und Klangfiguren wie gehäufte siLaute oder `männliche` Kadenzen (in der zitierten Strophe) lassen sich ebenfalls als akustische Annäherungen an Aspekte der Situation verstehen, die das Kind wahrnimmt. Wie Cwik formuliert: „Das Gedicht spricht nicht nur von einem akustischen Phänomen, sondern versucht dieses auf der materiellen Ebene der Wörter, aus denen es besteht, selbst wiederzugeben. $\ll^{12}$

Verschiedentlich ist betont worden, dass der (erfolgreiche) Gefühlsausdruck durch einen literarischen Text mit einem Lerneffekt für Rezipienten verbunden ist. Der skizzierten Theorie Steckers folgend, können wir anhand des Ausdrucks von Angst generalisierbares Wissen über ein wichtiges Charakteristikum von Angst erwerben; d.h., wir können (anhand eines Beispiels) lernen, was für eine angstvolle Weltwahrnehmung charakteristisch sein mag. So macht das Gedicht insbesondere deutlich, dass die ängstliche Person in ihrem

${ }^{11}$ Robert Stecker, Expression of Emotion in (Some of) the Arts, in: The Journal of Aesthetics and Art Criticism 42, 409-418, hier 410.

12 Cwik, Wie eine gespenstige Melodey, 279. 
Gefuihl, bedroht zu sein, konkrete Quellen der Bedrohung auf ihre Umwelt projiziert: Der "gespenstige Gräberknecht" und die "Spinnlenor«, so dürfen wir annehmen, stecken nicht wirklich hinter den Geräuschen im Moor - es handelt sich um Hirngespinste des sich ängstigenden Kindes. ${ }^{13}$

Während dieser Lerneffekt gleichsam rabstrakt und kaum literaturspezifisch ist - wir könnten dieses Wissen auch anhand eines psychologischen Traktats erwerben -, kann eine besondere Erkenntnisbedeutsamkeit literarischer Expressivität darin liegen, dass wir angesichts eines expressiven Kunstwerks unsererseits ein Gefühl dafür bekommen, wie es ist, in dem fraglichen Zustand zu sein. Dies ist sogenanntes qualitatives Wissen: Wir machen, wenn wir es erwerben, erstpersonale Bekanntschaft mit einer Gefühlsqualität, anstatt nur (gleichsam abstrakt) über Charakteristika des Gefühls belehrt zu werden. Man sollte es mit dieser These allerdings nicht übertreiben, und auch Jenefer Robinson meint vorsichtig "we are also shown something about what it is like to be in that state.$^{14}$ Wenn wir eine Idee davon bekommen, wie sich ein bestimmter Zustand anfühlt, dann sind wir insbesondere noch lange nicht selbst in diesem $\mathrm{Zu}-$ stand.Von einer Idee der Beklemmung, die jemand in seiner Angst fühlt, bis zur 'vollgültigen A Angst, die einem alles als bedrohlich erscheinen lässt, ist es schließlich ein weiter Weg. ${ }^{15}$

Dass uns ein literarisches Werk (vorübergehend) in den affektiven Zustand eines Protagonisten oder einer Protagonistin >versetzt (oder doch, wie beschrieben, eine erstpersonale Annäherung an gewisse qualitative Aspekte dieses Zustands gewährt), lässt sich als Ergebnis eines imaginativen Empathie-Prozesses beschreiben. Für Empathie ist charakteristisch, dass man sich (in seiner Vorstellung) in die Situation einer anderen Person versetzt. Das kann einerseits heißen, dass man simuliert, wie man selbst in der fraglichen Situation reagieren würde; andererseits kann es heißen, dass man zu simulieren versucht, wie sich die andere Person in der fraglichen Situation fühlen mag.

\footnotetext{
${ }^{13}$ Vgl.Thomas Wortmann, Schrecken ohne Ende, in: Interpretationen. Gedichte von Annette von Droste-Hülshoff, hg. v. Claudia Liebrand/Thomas Wortmann, Stuttgart 2014, 62-75, hier 64. Die Sekundärliteratur verweist in diesem Zusammenhang gern auf Goethes »Erlkönig", vgl. ebd. sowie Richard Alewyn, Die Literarische Angst, in: Aspekte der Angst, hg. v. Hoimar v. Ditfurth, Stuttgart 1965, 24-37, hier 35.

${ }^{14}$ Jenefer Robinson, Expression and Expressiveness in Art, in: Postgraduate Journal of Aesthetics (2007) 4; http://pjaesthetics.org/index.php/pjaesthetics/article/view/57 (22.05.2017), 30 .

${ }^{15} \mathrm{Vgl}$. zu entsprechenden Überlegungen Kendall L. Walton, Mimesis as Make-Believe. On the Foundations of the Representational Arts, Cambridge, MA 1990, Kap. 7.
} 
Für die literarische Rezeption ist die Empathie mit Figuren - also die (wie auch immer geartete) imaginative Simulation eines Verhaltens, das der beschriebenen Situation adäquat wäre - allerdings natürlich nicht der einzige Weg. Leserinnen und Leser können gleichsam `komplementäre Gefühle entwickeln angesichts der repräsentierten Gefühle der Figuren. Wenn sich ein Franz Moor an seiner Untat freut, bin ich meinerseits (hoffentlich) nicht erfreut, sondern vielleicht indigniert oder sogar entsetzt; die Liebe Romeos zu Julia erfüllt mich vielleicht mit Begeisterung (nicht aber meinerseits mit Liebe); der unangebrachte Stolz eines Protagonisten, den wir mögen, erfüllt uns mit (Fremd-)Scham. ${ }^{16}$ Auch Droste-Hülshoffs "Der Knabe im Moor» muss uns keineswegs ängstigen, weil wir uns in den Knaben einfühlen, von dem das Gedicht handelt; eine solche Identifikation mag uns sogar eher schwerfallen, wenn (und weil) wir nicht zum Gespensterglauben neigen und die Reaktion des Knaben übertrieben und kindisch anmutet. Bodo Plachta unterscheidet in seiner Interpretation von "Der Knabe im Moor" denn auch die "Angst" des Jungen vom "Gruseln", das bei Leserinnen und Lesern "aus[ge]löst « werde. ${ }^{17}$

Aber kann uns ein literarischer Text in Angst versetzen?

Bei »lovelybooks", einem sozialen Netzwerk für Literaturbelange, können Bücher als »deprimierend " getaggt werden, auf der Liste der solchermaßen ausgezeichneten Titel finden sich (auch) Klassiker der Weltliteratur, unter ihnen George Orwells »1984« (1949) oder Emily Brontës "Wuthering Heights" (1847).${ }^{18}$ Hier sind Leserinnen und Leser offenbar der Ansicht, dass ein bestimmtes Buch in eine bestimmte (>deprimierte`) Stimmung zu versetzen mag.

Bücher wie die genannten mögen entsprechende Effekte haben, weil es ihren Protagonisten nicht gut ergeht; wenn man Anteil genommen hat an den Geschicken einer Figur - wenn man sie vielleicht sogar liebgewonnen hat -, dann mag uns ihr Niedergang deprimieren.

Aber das ist nicht alles. Die genannten Werke zeichnen sich nicht nur dadurch aus, dass es sich um figurenbezogene $>$ Niedergangsgeschichten handelt; vielmehr zeichnen sie sich durch eine düstere ,Weltsicht aus: An der Geschichte der Protagonisten lässt sich eine

\footnotetext{
${ }^{16}$ Vgl. zur systematischen Unterscheidung dargestellter und eigener Gefühle Noël Carroll, A Philosophy of Mass Art, Oxford 1998, 260f.

${ }_{17}$ Bodo Plachta, DerVerlust des festen Bodens. Sozialgeschichtlicher und literarischer Kontext des Droste-Gedichts `Der Knabe im Moor`, in: Jahrbuch des Freien Deutschen Hochstifts 1997, 206-231, hier 227.

18 https://www.lovelybooks.de/stoebern/empfehlung/deprimierend/ (13.11.2019).
} 
bestimmte Weise ablesen, wie es sich mit dem menschlichen Leben im Allgemeinen verhält - und zwar, wohlgemerkt, nicht nur mit dem Leben der fiktiven Figuren, sondern mit unserem Leben. >Düster ist diese Weltsicht gewiss dann, wenn das Leben im Großen und Ganzen als leidvoll und das menschliche Streben als vergeblich, kurz: wenn die Existenz als sinnlos charakterisiert wird. Und während das spezifische Schicksal der Protagonisten uns nicht ereilen kann (weil sie erstens fiktiv sind und wir ihre ebenfalls fiktiven Bedrohungen nicht zu fürchten brauchen und weil wir zweitens nicht in just jenen kontingenten Umständen leben, wie sie die Welten von "1984 « und "Wuthering Heights" auszeichnen), so lassen sich allgemeine Aussagen oder Ansichten doch auf die eigene Situation abbilden, insofern diese Teil der allgemeinmenschlichen conditio ist.

Wenn man etwas wie dies als Quintessenz eines Werkes (als dessen >Botschaft oder >Lehre ) feststellt, so gibt es - idealtypisch - zwei Möglichkeiten: Man kann ein solches Buch aus der Hand legen und sich anderen, freudvolleren Dingen zuwenden. Ist man dann wieder aufgeheitert, so ist die kurze Episode niedergeschlagener Stimmung vorbei. Das Buch mag >deprimierend sein, aber man hat sich davon nicht anstecken lassen. Auch dass man wirklich in einen Zustand der Angst versetzt wurde, wird man nicht sagen wollen.

Die andere Möglichkeit könnte so aussehen: Man nimmt die Aussage des Textes ernst; man hält sie für eine wahre Beschreibung des Lebens im Allgemeinen; man identifiziert sich vielleicht sogar mit ihr (weil man sie nämlich für wahr hält), d.h., man realisiert, dass das Gesagte auch für das eigene Leben gilt (und eben nicht nur für das Leben sim Allgemeinen ).

Natürlich können literarische Werke in dieser Weise wirken. Peter Kivy hebt hervor, dass die Lektüre von "serious novels" ein 'Nachleben` ("afterlife«) hat:

It is a period subsequent to the completion of the novel during which the images and content linger on in the mind to be savored and thought about. A literary experience, where it is of a serious novel, that lacks this postpartum period of contemplation, lacks something that is, I suggest, an integral part of the full literary experience. ${ }^{19}$

Ein Buch, das einen Reflexionsprozess und den Erwerb neuer Überzeugungen anstößt, kann gleichsam aufrütteln. Kafka beschreibt das in einem Brief mit recht dramatischen Ausdrücken:

${ }_{19}$ Peter Kivy, The Performance of Reading, Malden, MA, u.a. 2006, 108. 
Wenn das Buch, das wir lesen, uns nicht mit einem Faustschlag auf den Schädel weckt, wozu lesen wir dann das Buch? Damit es uns glücklich macht, wie du schreibst? Mein Gott, glücklich wären wir eben auch, wenn wir keine Bücher hätten, und solche Bücher, die uns glücklich machen, könnten wir uns zur Not selber schreiben. Wir brauchen aber die Bücher, die auf uns wirken wie ein Unglück, das uns sehr schmerzt [...]. ${ }^{20}$

Bücher, die in Kafkas Sinne "wie ein Unglück [wirken] «, verändern etwas an den Überzeugungen ihrer Leser; sie mögen dazu führen, dass man die Welt nun mit anderen Augen sieht. Ein Beispiel für einen solchen Text, der im Sinne Kivys "serious" (und im Sinne Kafkas "ein Unglück«) sein dürfte, ist Tolstojs "Der Tod des Iwan Iljitsch" (russ. 1886). Erzählt wird das Leben des Petersburger Richters Iwan Iljitsch, und zwar proleptisch beginnend mit einer Episode nach seinem Tod und der Ankündigung, seine "Lebensgeschichte" sei "sehr einfach und sehr gewöhnlich und doch entsetzlich «. ${ }^{21}$ Teils stark raffend, teils en détail wird erzählt, wie es mit Iwan Iljitschs Leben zu Ende geht. Der Protagonist erkrankt und muss mit fortschreitender Krankheit erkennen, dass alles, was ihm im Leben Freude machte, nun nichts mehr bedeutet; dass seine Freuden auch zu Lebzeiten hohl und nichtig waren; dass seine Familie (fast ausnahmslos) nicht zu ihm steht, jetzt, wo er krank und hilflos ist (ja, dass er seine Familie vielmehr mit seinem Kranksein belastet); und vor allem, dass sein baldiger Tod unausweichlich ist und nichts weniger als seine Auslöschung bedeutet. Iwans Qualen werden drastisch geschildert, in der Tat: sie sind »entsetzlich". - Was lehrt dieser Text? "Der Tod des Iwan Iljitsch" wirft zunächst einmal die Frage auf, wie Iwans Leben und Sterben zu beurteilen ist: Ist sein Leben ebenso "gewöhnlich" wie sinnlos? Führt sein unmittelbar bevorstehender Tod Iwan zuletzt zu der Einsicht, dass er sein Leben falsch gelebt und mithin vergeudet hat? Sind diese letzten Momente der Einsicht am Ende sogar geeignet, wo nicht seine Fehler aufzuwiegen, so doch wenige, letzte Momente eines gelingenden Lebens zu realisieren?22

\footnotetext{
${ }^{20}$ Franz Kafka, Briefe 1902-1942, hg. v. Max Brod unter Mitarbeit v. Klaus Wagenbach, Frankfurt a.M. 1975, 27f.; hier zitiert nach Thomas Anz, Praktiken und Probleme psychoanalytischer Literaturinterpretation - am Beispiel von Kafkas Erzählung Das Urteil, in: Kafkas "Urteil" und die Literaturtheorie. Zehn Modellanalysen, hg. v. Oliver Jahraus/Stefan Neuhaus, Stuttgart 2002, 126-151, hier 142.

${ }^{21}$ Leo N. Tolstoj, Der Tod des Iwan Iljitsch, in: Ders., Die großen Erzählungen, aus d. Russ. übers. v. Arthur Luther/Rudolf Kassner, München 1997, 11-82, hier 21.

${ }^{22}$ So Frances M. Kamm, Rescuing Ivan Ilych: How We Live and How We Die, in: Ethics 113 (2003), 202-233.
} 
Für Gerald Lang sind diese Fragen zu verneinen, und sie verweisen darüber hinaus auf allgemeine Lehren über das Leben und den Tod. Langs gewiss düsterer Lesart des Textes zufolge lassen sich die Lehren des Romans so zusammenfassen:

Ivan's way of coping with his death does not make him a better person, but merely exposes and magnifies what was always objectionable about him. Alas, and moreover, Ivan's case enjoys potential relevance to us all, even if we are not bound to repeat Ivan's precise mistakes. [My conclusions are] that the manner of death does not easily restore, or compensate for, bad lives, and that good lives do not easily prepare us for death. Whether we have led a good life or a bad life, death is likely to present us with problems of reconciliation. ${ }^{23}$

Und weiter:

Death is tacked on to our lives in ways which make it very difficult for us to achieve reconciliation with it, or to prepare adequately for it. In this sense, Ivan's case, depicted with such deft particularity by Tolstoy, has universal resonance. Each of us will have different projects to try to complete, different memories to fall back on, and different people to rely upon and say our farewells to, if and when we come to know in advance that we are dying. But the predicament we will be in may well be closer to that of a fictional judge in nineteenth-century Saint Petersburg than we might have been inclined to think.

Langs Interpretation des Romans, man mag sie teilen oder nicht, macht den suniversalen Anspruch des Romans deutlich: Was ausgesagt wird, soll ernst genommen - und das heißt: von Leserin und Leser auf das eigene Leben bezogen - werden. Wenn die >Botschaft des Romans düster ist, wie von Lang für Tolstojs Erzähltext angenommen, und wenn sie als wahr erkannt wird - kann der Text seine Leserinnen und Leser dann in Angst versetzen?

Vielleicht. Wir müssen die Frage hier offenlassen. In jedem Fall wird man sagen können, dass Tolstojs Erzählung verschiedene Dimensionen >literarischer Angst in sich vereint: Dargestellt wird die Angst des Protagonisten angesichts seines Todes; ferner wird diese Angst auch ausgedrückt im Sinne Steckers, denn der Roman führt uns vor Augen, wie sich die Welt aus den Augen jemandes ausnimmt, der wie Iwan von fürchterlicher Angst gequält wird, und bei der

${ }^{23}$ Gerald Lang, What Does Ivan Ilyich Need To Be Rescued From?, in: Philosophy 89 (2014), 325-347. 
Lektüre mögen wir ein (und sei es vages) Gefühl dafür bekommen, wie es ist, diese Angst zu erleben. Und schließlich hat der Roman das Potenzial, ein »Unglück« in Kafkas Sinne zu sein: Das Buch möchte auf unsere Überzeugungen wirken, wie zu leben richtig ist, und wenn es das tut, dann könnte das eine Stimmung auslösen, die als Angst vielleicht nicht ganz falsch beschrieben ist - und die erst dann wieder vergeht, wenn der Überzeugung von der Entsetzlichkeit des menschlichen Schicksals etwas Wirksames entgegengesetzt wird. ${ }^{24}$

Eine solche Charakterisierung des Romans mag in ihrer Einseitigkeit nun freilich unbefriedigend wirken: Denn ist "Der Tod des Iwan Iljitsch" nicht in erster Linie ein Roman - ein literarisches Kunstwerk also, das als solches nicht (allein) für die Einsichten geschätzt werden sollte, die es vermitteln mag oder nicht, sondern das vielmehr in erster Linie ästhetisches Vergnügen bereiten sollte?25 Und hier schließt sich die Frage an:Wie kann die drastische Schilderung des entsetzlichen Todes Iwan Iljitschs überhaupt so etwas wie Vergnügen bereiten?

Die Frage nach dem "Grund des Vergnügens an tragischen Gegenständen" - so die Formulierung eines einschlägigen Traktats von Friedrich Schiller aus dem Jahr 1792 - hat das Nachdenken über die Tragödie im Besonderen und die Darstellung des Schrecklichen

\footnotetext{
${ }^{24}$ Dante Andrea Franzetti hebt in seinem Text »Kleines Traktat von der Angst« hervor, dass Literatur Gefühle stets nur abgeschwächt kommunizieren könne, weil das Dargestellte schließlich stets sprachlich vermittelt sei; das unterscheide Literatur von einem Bild wie Munchs berühmtem "Schrei«, dessen "Darstellung der Angst [...] durch keinen Text überboten werden" könne (Dante Andrea Franzetti, Kleines Traktat von der Angst, in: Das helle und das dunkle Zimmer. Schweizer Schriftstellerinnen und Schriftsteller schreiben von der Angst, hg. v. Renate Nagel, Zürich/Frauenfeld 1988, 220-231, hier 220). Das entspricht einerseits dem oben Angedeuteten: Wird (etwa durch ein Gedicht) ein Gefühl ausgedrückt, so bekommen wir (allenfalls) eine Idee davon, wie es sich anfühlt, das Gefühl zu haben. (Die Frage wäre, ob sich dies bei Werken der Malerei anders verhält?) Andererseits ist hier die besondere Befähigung der Literatur (im Unterschied zur Malerei) als Quelle eines Überzengungserwerbs oder -wandels hervorzuheben. Just diese Eignung befähigt Literatur in besonderer Weise, die Welt mit anderen Augen sehen zu lassen, und darin mag ebenfalls eine besondere Befähigung liegen, Stimmungen dauerhaft zu fundieren.

${ }^{25}$ Jerrold Levinson deutet eine entsprechende Verbindung zwischen der Wertschätzung eines Kunstwerkes als Kunstwerk (im Unterschied zu einer Wertschätzung unter anderen Gesichtspunkten, etwa als Quelle von Wissen) und dem ästhetischen Vergnügen an: "Without a distinction between the aesthetic pleasure in an artwork and other pleasures to be had from it, it is unlikely one will be able to explain satisfactorily what the proper appreciation of art - that is, its appreciation as art - might consist in." (Jerrold Levinson, The Pleasures of Aesthetics. Philosophical Essays, Ithaca/London 1996, 3). Und: "The arts are intended, as much as anything, to give pleasure and are, by and large, well suited to provide it.« (Ebd., 11)
} 
in der Kunst im Allgemeinen seit der Antike beschäftigt und ist in der Forschungsliteratur gut dokumentiert. ${ }^{26}$ Bei genauerem Hinsehen zeigt sich, dass hier eine ganze Reihe miteinander verbundener Probleme in Rede steht. So wurde u.a. für erklärungsbedürftig gehalten, dass Rezipienten positive Gefühle (Vergnügen) angesichts negativer Gegenstände (jemandes Leid) entwickeln; dass Rezipienten sich freiwillig Situationen aussetzen, in denen sie 'Zeuger von jemandes massivem Leid werden (also z.B. ein Buch lesen, das einen >deprimierenden< Gehalt hat, s.o.); dass sie (moralisch) billigen, auf (die Darstellung von) jemandes Leid mit Vergnügen zu reagieren; und dass eine Kunstform (wie die Tragödie) hochgeschätzt wird, für die die Darstellung von jemandes schwerem Leid wesentlich ist. - Alle diese Probleme scheinen die literarische Darstellung von Angst unmittelbar zu betreffen und fragwürdig zu machen. - Wenn eingangs gesagt wurde, es nehme nicht wunder, dass die Angst als ein allgemeinmenschliches Problem Gegenstand der sschönen Literatur ist, so zeigt sich nunmehr, dass diese Aussage in einer bestimmten Hinsicht der Erläuterung bedarf: dann nämlich, wenn die sschöne Literatur als Kunstform angesehen wird, die ihren Daseinszweck nicht zuletzt darin hat, ästhetisches Vergnügen hervorzurufen.

In der Geschichte von Poetik und Ästhetik sind viele verschiedene Versuche zur Lösung der genannten Probleme vorgeschlagen worden. Die aktuellere Forschung wiederum hat neue Lösungen entwickelt und die bestehenden zu systematisieren versucht. Jerrold Levinson unterscheidet verschiedene Ansätze, denen die vielen Antworten zugeordnet werden können: ${ }^{27}$,Kompensatorischer Theorien gehen davon aus, dass es Aspekte der (komplexen) Erfahrung negativer Gefühle gibt, die den negativen Charakter dieser Gefühle kompensieren; >Konversionstheorien gehen davon aus, dass sich der negative Charakter einschlägiger Gefühle im Rezeptionsakt in einen positiven verwandelt; >Organizitäts-Theorien halten die Negativität

\footnotetext{
${ }^{26}$ Vgl. die Überblicksdarstellungen in Jerrold Levinson, Introduction, in: Suffering Art Gladly. The Paradox of Negative Emotion in Art, hg. v. Jerrold Levinson, Basingstoke/New York 2014, x-xvi; vgl. auch Thomas Anz, Angst und Ekel. Über UnlustGefühle und ihre ästhetische Genießbarkeit beim Lesen, in: Figurationen 15.2 (2014), 52-65; speziell zum 18. Jh. vgl. auch Carsten Zelle, Über den Grund des Vergnügens an tragischen Gegenständen in der Ästhetik des achtzehnten Jahrhunderts (mit einem bibliographischen Anhang), in: Schönheit und Schrecken. Entsetzen, Gewalt und Tod in alten und neuen Medien, hg. v. Peter Gendolla/Carsten Zelle, Heidelberg 1990, 55-91.

${ }^{27}$ Vgl. knapp zusammenfassend Levinson, Introduction, insbes. xii, sowie die weiteren Beiträge in diesem Band; für ein alternatives Ordnungsschema vgl. Anz, Angst und Ekel.
} 
der in den Rezeptionsakt involvierten Gefühle für einen wesentlichen (’organischen`) Bestandteil der positiven Gesamterfahrung der Kunstrezeption; >revisionistischer Theorien besagen, dass snegativer Gefühle mitnichten intrinsisch unangenehm oder unerwünscht sind (sondern in bestimmten Kontexten sogar als angenehm erfahren werden können); und `Deflationstheorien ‘ bestreiten schlicht, dass in der (Tragödien-)Rezeption tatsächlich (vollgültige, eigentliche) negative Gefühle hervorgerufen werden.

Alle diese Theorien verfügen über ein gewisses Erklärungspotenzial im Hinblick auf die oben aufgeworfenen Probleme. Und sie können auch zu erklären helfen, weshalb Angst als Objekt auch literarischer-künstlerischer Darstellung existiert. In Bezug auf die Angst scheinen vor dem Hintergrund des Gesagten zunächst einmal >deflationäre Theorien eine gewisse Erklärungskraft zu haben: In Fällen nämlich, in denen das Buch kein Kafka'sches "Unglück" ist (das Werk also keinen nachhaltigen Überzeugungswandel hervorruft, der derart ist, dass er Angst fundieren könnte), löst das Buch bei seinen Leserinnen und Lesern ganz einfach keine (intrinsisch unangenehme) Angst aus. Ausgelöst wird in diesen Fällen - und hier könnte es sich durchaus um die Mehrzahl der Fälle handeln - vielmehr allenfalls ein leichtes Gruseln, das (und hier mögen rrevisionistische`Theorien anklingen) als intrinsisch angenehm erfahren wird. Für jene (seltenen?) Fälle echter Angst, die Rezipienten als Resultat der Lektüre empfinden mögen, könnte ferner rundheraus bestritten werden, dass dies etwas mitVergnügen zu tun habe. Dieser Aspekt des Lektüreakts (bzw. von dessen "reflective afterlife« im Sinne Kivys) mag schlicht unangenehm sein (so dass es mithin auch kein erklärungsbedürftiges Vergnügen zu konstatieren gibt).Vereinbar damit ist natürlich, dass andere Aspekte des Rezeptionsaktes angenehm bzw. im ästhetischen Sinne vergnüglich wahrzunehmen sein mögen, wie >Kompensationstheorien` und auch `Organizitätsı-Theorien es vorhersagen. So besticht etwa Tolstojs »Der Tod des Iwan Iljitsch« durch eine ausgeklügelte Erzählstruktur; und auch die Anschaulichkeit der Darstellung oder deren schlichte Wucht mögen, für sich genommen, Gegenstand von Bewunderung sein und ästhetisches Vergnügen fundieren..$^{28}$

- Dr. Tilmann Köppe ist Professor für Analytische Literaturwissenschaft am Seminar für Deutsche Philologie der Universität Göttingen. Zu seinen Arbeitsgebieten gehören insbesondere die Literaturtheorie und die philosophische Ästhetik.

${ }^{28}$ Ich danke Anna Ertel für Hinweise zu einer früheren Fassung dieses Beitrags. 